REFERENCES:

[1] Varga J, Abraham D. Systemic sclerosis: a prototypic multisystem fibrotic disorder. J Clin Invest 2007;117:557-67.

[2] Marchwicka A, Cebrat M, Łaszkiewicz A, Snie ' zewski Ł, Brown G, Marcinkowska $E$. Regulation of vitamin $D$ receptor expression by retinoic acid receptor $\alpha$ in acute myeloid leukemia cells. J. Steroid Biochem. Mol. Biol. 2016;159:121-130.

[3] Zerr P, et al. Ann Rheum Dis 2014;0:1-8. doi:10.1136/annrheumdis-2013204378

Acknowledgements: This research carried out at Dokuz Eylul University Medicine Faculty of Research Laboratory (R-LAB).

Disclosure of Interest: None declared

DOI: 10.1136/annrheumdis-2018-eular.2158

\section{AB0184 DIAGNOSTIC IMPACT IN THE CLINICAL SETTING OF NAILFOLD VIEOCAPILLAROSCOPY ON CONNECTIVE DISEASES}

C. Soleto, L. Torrens, J.C. Nieto González, on behalf of Claudia Sáenz Tenorio, Ana M. Anzola, Liz. Rocío Caballero Motta, Leticia. García, María Correyero. Plaza, D.D.D. González Benítez, Belén Serrano. Benavente, Julia Martínez. Barrio, Javier. López-Longo, Alfonso. Ariza, Indalecio Monteagudo. Sáez. Rheumatology, Hospital General Universitario Gregorio Marañón (H.G.U.G.M.), Madrid, Spain

Background: Nailfold video-capillaroscopy (NVC) is a non-invasive technique that allows visualisation of structure and distribution of capillaries at the nailfold level ${ }^{1}$, altered somehow in some connective diseases, specially in the Scleroderma (Scl) disease spectrum. The main indication of this technique is the investigation of Raynaud's phenomenon (RF).

Objectives: Our objective is to investigate the diagnostic impact of NVC in the daily clinical practice.

Methods: The design is an observational, longitudinal, retrospective and descriptive study, which included patients with at least one NVC between June 2012 and December 2016 from our Rheumatology register of patients. We collected demographic data (age, gender, indication, autoantibodies, etc.), number of explorations performed and their result. We also collected in a dichotomist fashion if the NVC contributed in the diagnostic workup, between one consultation and the other after the NVC realisation.

Results: 437 patients were included with a total of 637 explorations. Of these 437 patients, $115(24.1 \%)$ had a second NVC, $39(8.2 \%)$ a third one, $9(1.9 \%)$ a fourth and only two with a fifth NVC (both with diagnostic of Scl). We noticed a diagnostic change between the first consultation and the next one in 35 cases (5.49\%). In 14/ $35(40 \%)$ of these cases, the NVC played an important role in the diagnostic change, with changes in the NVC pattern, from normal or unspecific to Scl pattern (table 1). These changes, occurred after the first NVC in 10 patients $(71.4 \%), 3$ (21.4\%) after the second, and $1(7.1 \%)$ after the third exploration in addition of new disease manifestations, diagnostic tests and other image techniques. Of these 14 patients, $100 \%$ had positive ANA, 5 (35.7\%) Anticentromere Antibodies (Ab), 1 (7.1\%) anti-Ro Ab and 1 (7.1) Antiphospholipid Ab.

\begin{tabular}{lcc} 
Abstract AB0184 - Table $\mathbf{1}$ & \\
\hline Change & $\mathrm{n:14}(\%)$ & Pattern \\
\hline RF to Scl & $11(78.5 \%)$ & Early $7 / 11(63.6 \%)$ \\
& & Active $2 / 11(18.1 \%)$ \\
UC to Scl & Late $2 / 11(18.1 \%)$ \\
RF to UC & $2(14.2 \%)$ & Early $2(100 \%)$ \\
& $1(7.1 \%)$ & Early $1(100 \%)$ \\
\hline
\end{tabular}

Raynaud phenomenon (RF); Scleroderma (Scl); Undifferentiated connective disease (UC); Mixed connective tissue disease (MCTD); Systemic Lupus Erythematosus (SLE); primary Sjögren syndrome (pSS).

Of the 21 patients with a diagnostic change who did not developed a Scl pattern we have:

Normal pattern: $1 \mathrm{RF}$ to possible Scl and $1 \mathrm{RF}$ to UC.

Limit of normality: 1 SLE to MCTD, 4 RF to UC, 1 RF to $p S S, 1$ RF to MCTD and 1 UC to MCTD.

Unspecific (mild): 3 RF to UC, 2 RF to Scl and $1 \mathrm{UC}$ to overlap syndrome. Unspecific (moderate): $1 \mathrm{RF}$ to $\mathrm{UC}, 1 \mathrm{RF}$ to $\mathrm{Scl}, 1 \mathrm{UC}$ to MCTD and $1 \mathrm{RF}$ to MCTD.
Conclusions: The NVC in our centre had a limited but important impact in the diagnostic process of connective diseases. This impact was specially relevant in patients diagnosed with Scleroderma. The probability of having a diagnostic change diminishes with successive explorations.

\section{REFERENCE:}

[1] Cutolo M, Pizzorni C, Secchi ME, Sulli A. Capillaroscopy. Best Pract Res Clin Rheumatol 2008 Dec;22(6):1093-108.

Disclosure of Interest: None declared DOI: 10.1136/annrheumdis-2018-eular.6720

\begin{tabular}{|l|l|}
\hline AB0185 & ALTERED EXPRESSION OF RELAXIN RECEPTOR \\
RXFP1/LGR7 IN DERMAL FIBROBLASTS CONTRIBUTES \\
TO THE INEFFICACY OF RELAXIN-BASED ANTI- \\
FIBROTIC TREATMENTS IN SYSTEMIC SCLEROSIS
\end{tabular}

C. Corallo ${ }^{1}$, M. Cutolo ${ }^{2}$, S. Soldano ${ }^{2}$, S. Cheleschi ${ }^{3}$, A. Fioravanti ${ }^{3}$, A.M. Pinto ${ }^{4}$, A. Renieri' ${ }^{4}$, R. Nuti' ${ }^{1}$, N. Giordano'. 'S Scleroderma Unit, Department of Medicine, Surgery and Neurosciences, University of Siena, Siena; ${ }^{2}$ Research Laboratory and Academic Division of Clinical Rheumatology, Department of Internal Medicine, University of Genoa, Polyclinic San Martino Hospital, Genoa; ${ }^{3}$ Rheumatology Unit, Department of Medicine, Surgery and Neurosciences; ${ }^{4}$ Medical Genetics, Department of Medical Biotechnologies, University of Siena, Siena, Italy

Background: Systemic Sclerosis (SSc) is an autoimmune disease characterised by progressive fibrosis of the skin and internal organs, coupled to widespread vascular pathology. ${ }^{1}$ The pathogenesis is still poorly understood and there is no effective treatment for the fibrotic process. ${ }^{1}$ Relaxin is a potent anti-fibrotic hormone that has been tested in the past to ameliorate skin, lung and kidney fibrosis in SSc but the results remain controversial. ${ }^{2}$

Objectives: The aim of the study is to evaluate the presence of mutations in RXFP1 gene (encoding the relaxin receptor RXFP1/LGR7), and to assess mRNA and protein levels of the receptor in dermal fibroblasts of SSc patients, in order to understand the clinical inefficacy of relaxin-based anti-fibrotic treatments in the disease.

Methods: Fibroblasts were isolated from unaffected and affected skin samples of $(n=20)$ of limited cutaneous SSc (LcSSc) and from $(n=20)$ affected skin of diffuse cutaneous SSc (DcSSc) patients. Fibroblasts derived from healthy subjects were used as controls. Sequencing of exonic target regions of interest for gene RXFP1 was performed coupled with mRNA transcript variant analysis. RXFP1/LGR7 mRNA and protein levels were assessed by quantitative-real-time-PCR (qPCR) and by immunocitochemistry (ICC) in cultured SSc and healthy fibroblasts Finally, synthesis of collagen and alpha-smooth-muscle actin (a-SMA) of transforming-growth-factor-beta-1 (TGF- $\beta 1$ ) induced fibroblasts were assessed after 24 hours pre-treatment with serelaxin (a recombinant form of human relaxin-2 targeting the relaxin receptor RXFP1/LGR7).

Results: Sequencing of RXFP1 gene showed no relevant (single nucleotide polymorphisms) SNPs or small insertions and deletions (InDels) in affected LcSSc/ DcSSc fibroblasts. No relevant mutations were found in unaffected LcSSc and healthy fibroblasts as well. However, alternatively spliced transcript variants encoding multiple isoforms were observed for this gene in all the fibroblast populations. The total RXFP1 mRNA levels resulted upregulated $(p<0.05)$ in the affected LcSSc/DcSSc fibroblasts compared to unaffected LcSSc $(p<0.05)$ and to healthy ones $(p<0.05)$. On the contrary, ICC demonstrated the absence of RXFP1/LGR7 receptor in affected LcSSc/DcSSc fibroblasts and the regular expression in unaffected LcSSc and healthy fibroblasts. In fact, serelaxin pre-incubation was unable to counteract the TGF- $\beta 1$ driven upregulation of collagen and a-SMA in affected LcSSc/DcSSc fibroblasts only, but not in unaffected LcSSc and healthy ones.

Conclusions: The absence/altered expression of relaxin receptor RXFP1/LGR7 in the affected fibroblasts of SSc patients could explain the inefficacy of relaxinbased anti-fibrotic treatments in the disease. The exclusion of RXFP1 gene mutations could lead to the hypothesis that the presence of receptor splice variants could exert a dominant negative effect on the wild type isoform in terms of maturation, and subsequent trafficking to the cell surface, resulting in loss of function.

\section{REFERENCES :}

[1] Denton CP. Advances in pathogenesis and treatment of systemic sclerosis. Clin Med (Lond) 2016;16:55-60.

[2] McVicker BL, Bennet RG. Novel Anti-fibrotic therapies. Front Pharmacol 2017;8:318.

Disclosure of Interest: None declared DOI: 10.1136/annrheumdis-2018-eular.7153 